\title{
A new saurolophine hadrosaurid (Dinosauria: Ornithopoda) from the Upper Cretaceous of Shandong, China
}

\author{
JIALIANG ZHANG ${ }^{1,2}$, XIAOLIN WANG ${ }^{1,2^{*}}$, QIANG WANG ${ }^{1}$, SHUNXING $^{2}$ \\ JIANG ${ }^{1}$, XIN CHENG ${ }^{1}$, NING LI $^{1}$ and RUI QIU ${ }^{1,2}$ \\ ${ }^{1}$ Key Laboratory of Vertebrate Evolution and Human Origins of the Chinese Academy of Sciences, Institute of Vertebrate \\ Paleontology and Paleoanthropology, Chinese Academy of Sciences, PO Box 643, Beijing, 100044, China \\ ${ }^{2}$ University of Chinese Academy of Sciences, Beijing, 100049, China \\ Manuscript received on January 6, 2017; accepted for publication on April 4, 2017
}

\begin{abstract}
How to cite: ZHANG J, WANG X, WANG Q, JIANG S, CHENG X, LI N AND QIU R. 2019. A new saurolophine hadrosaurid (Dinosauria: Ornithopoda) from the Upper Cretaceous of Shandong, China. An Acad Bras Cienc 91: e20160920. DOI 10.1590/0001-3765201720160920.
\end{abstract}

\begin{abstract}
A new saurolophine hadrosaurid, Laiyangosaurus youngi gen. et sp. nov. is described and phylogenetically analyzed based on several cranial elements from the Jingangkou Formation, Wangshi Group, Upper Cretaceous of Laiyang, Shandong, China. Laiyangosaurus youngi differs from other members of the saurolophine clade on the basis of a number of autapomorphies, including a prominent and narrow ridge on the lateral side of the nasal which forms the posterodorsal and posterior margin of the circumnasal depression, a primary ridge that runs along most of the maxillary tooth row that is slightly deflected posteriorly, a retroarticular process of the surangular that is dorsolateroposteriorlly recurved, and orbital margins that are wider than the infratemporal margins of the jugal. This new taxon can be further distinguished by a number of unique combination of characters, including dorsal margin of nasal is flat, absence supracranial crest, a relatively shallow and rostrodorsally directed caudal margin of the lacrimal process of the jugal, and one or more foramina present on the rostral surface of the premaxilla. A phylogenetic analysis indicates that $\mathrm{L}$. youngi comprises a monophyletic clade, which is known as Edmontosaurini.
\end{abstract}

Key words: Upper Cretaceous, Wangshi Group, Laiyangosaurus youngi, Saurolophinae, Shandong, China.

\section{INTRODUCTION}

Hadrosauroids were diverse and abundant large herbivorous dinosaurs that lived during the Cretaceous (You et al. 2003, Horner et al. 2004, Prieto-Márquez 2010a, Wang et al. 2013b). This lineage of dinosaurs includes some basal forms,

Correspondence to: Xiaolin Wang

E-mail: wangxiaolin@ivpp.ac.cn

ORCid: http://orcid.org/0000-0003-2205-2103

* Contribution to the centenary of the Brazilian Academy of Sciences. also known as non-hadrosaurid hadrosauroids, and the monophyletic Hadrosauridea (PrietoMárquez 2010b, Wang et al. 2013b). Hadrosaurids are characterized by duck-like bills and complex dentitions organized into dental batteries (Horner et al. 2004); many species had hypertrophied nasal passages associated with a wide variety of supracranial crests, the nature of which forms the basis of their classification (Ostrom 1962, Hopson 1975, Horner et al. 2004, Prieto-Márquez 2010b). Studies have shown that the Hadrosauridae can be 
divided into two main clades, the Saurolophinae comprising flat-headed or solid-crested hadrosaurids, and the Lambeosaurinae, comprising the hollow-crested hadrosaurids (Horner et al. 2004, Prieto-Márquez 2010b). Hadrosauroids were widespread in their distribution between the Aptian and late Maastrichtian; their fossil remains have been found in Asia, Europe, North and South America, Africa, and Antarctica (Lund and Gates 2006, Prieto-Márquez 2010a, Mateus et al. 2012). Indeed, to date, a number of fossil hadrosauroids have been found in China (Lund and Gates 2006, Wang et al. 2013b, Xing et al. 2014a), in particular from the Laiyang Basin in Shandong (Wang et al. 2010, 2012).

The Laiyang Basin is located in eastern Shandong Province and has yielded the abundant fossil remains of plants, insects, and vertebrates, especially dinosaurs and their eggs (Fig. 1) (Wang et al. 2010, 2012, 2013a). The terrestrial Cretaceous strata in this basin comprise the Early Cretaceous Laiyang and Qingshan groups, as well as the Late Cretaceous Wangshi Group, which comprises the Xingezhuang, Jiangjunding, Jingangkou, and Changwangpu formations (Hu et al. 2001). To date, all Laiyang hadrosauriods have been collected from the Jiangjunding and Jingangkou formations (Wang et al. 2010, 2013a); Tan (1923) was the first to collect dinosaur bones from the Jiangjunding Formation at Jiangjunding in Laiyang, while later, Wiman (1929) described some of these specimens which was named Tanius sinensis, in honor of the collector. Later, Young (1958) named a new member of Lambeosaurinae, Tsintaosaurus spinorhinus, and erected a second species of Tanius, T. chingkankouensis, based on material collected from the Jingangkou Formation in Jingangkou, Laiyang. In 1964, a number of other large hadrosaurid bones were collected from the Wangshi Group of Laiyang and Zhucheng; these materials were later studied and named Shantungosaurus giganteus (Hu 1973, Hu et al. 2001). In 1976, the third species of Tanius, T. laiyangensis, was erected on the basis of a sacrum and right ilium found in the same place as Tsintaosaurus (Zhen 1976). In addition of hadrosauroid material, the Wangshi group in Laiyang has yielded a number of other dinosaurs (Young 1958, Dong 1978, Buffetaut and Tong 1995), four families, five genera, and eleven species of dinosaur eggs (Wang et al. 2010), as well as a turtle egg (Wang et al. 2013a). These dinosaur fossils and eggs are referred to as the Laiyang Hadrosauroid Fauna and Dinosaur Egg Fauna (Wang et al. 2010, 2012).

Since 2010, our team has been re-excavating at Locality 1 where Tsintaosaurus was first found. We discovered Locality 2, $1 \mathrm{~km}$ east to the Locality 1 (Fig. 1); during these excavations, we found many dinosaurs as well as their eggs, alongside turtles, their eggs, crocodile teeth, gastropods, and plant fragments in Upper Cretaceous Jingangkou Formation sediment at these two localities. More recently, Yan \& Chen (2005) obtained an isotope age of $73 \mathrm{Ma}$ for the Jingangkou Formation (which they called the Hongtuya Formation) based on a basalt sample from the town of Daxizhuang in Jiaozhou City. It is noteworthy that the strata within Locality 2 comprise pebbly sandstone and siltstone that derived from mudflows and flood plain facies. The gravels at this site comprise poorly-sorted siltstones and sandstone, while the long bones lie oblique crossed, or perpendicular, to the bone bed layer. These typical taphonomic characteristics of mudflow indicate that when alive this group of animals were swallowed and torn by a sudden flood and mudflow, bodies were carried a short distance and buried rapidly, and the fragments were not distantly separated. All the fossils described in this paper, collected from Locality 2 at Laiyang, belong to five individuals (two large ones and three small ones), since each individual encapsulated within a small area. They display typical saurolophine morphologies, and the same positional elements 


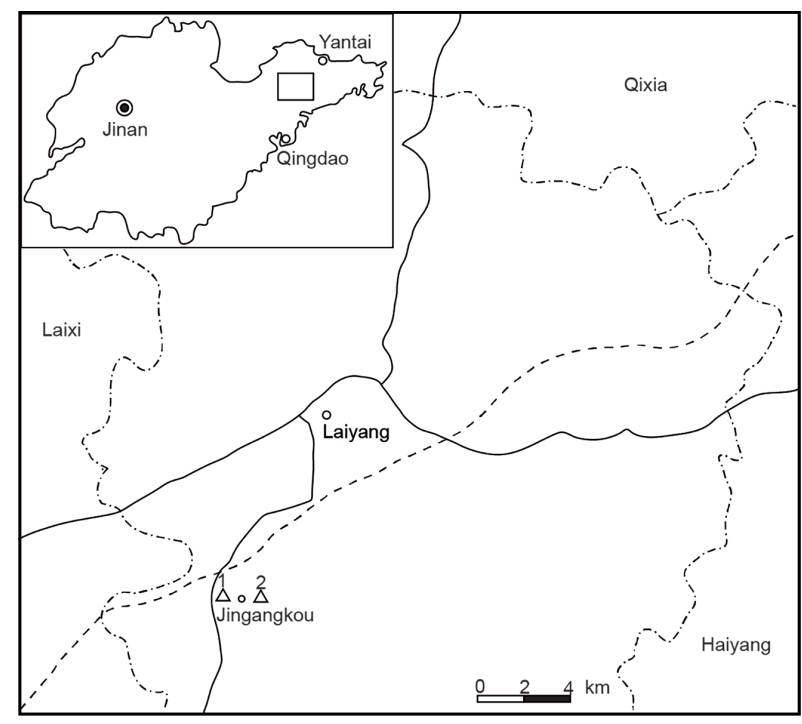

Figure 1 - Locality map and stratigraphic column for Laiyangosaurus. Locality 1 of Tsintaosaurus and locality 2 of Laiyangosaurus in Laiyang, Shandong, China (Modified from Wang et al. 2012).

share similar features. Thus, these hadrosaurid skull bones can be diagnosed as a new genus and species.

\section{SYSTEMATIC PALEONTOLOGY}

Ornithischia Seeley, 1887

Ornithopoda Marsh, 1881

Hadrosauroidea Cope, 1869

Hadrosauridae Cope, 1869

Saurolophinae Brown, 1914 (sensu Prieto-Márquez 2010b)

\section{Laiyangosaurus gen. nov.}

Etymology: laiyang, referring to the city where the fossils discovered; saurus, the transliterated Greek work 'sauros', referring to lizard.

Type species: Laiyangosaurus youngi, sp. nov.

Diagnosis: As for the species.

Laiyangosaurus youngi, sp. nov.

Etymology: youngi, in commemoration of the 120th Anniversary of Dr. Chungchien Young's Birth. Dr. Young is the pioneer of vertebrate paleontological research in Laiyang, and who has discovered many dinosaurs at this locality.
Holotype: IVPP V 23401, consisting of a large left maxilla, IVPP V 23401.1; a right squamosal, IVPP V 23401.2; and a left dentary, IVPP V 23401.3; housed in the Institute of Vertebrate Paleontology and Paleoanthropology, Chinese Academy of Sciences, Beijing, China.

Referred specimens: IVPP V 23402, consisting of a small left maxillae, IVPP V 23402.1; a small right premaxilla, IVPP V 23402.2; a small right nasal, IVPP V 23402.3; a small left squamosal, IVPP V 23402.4; a small right dentary, IVPP V 23402.5, a small left dentary, IVPP V 23402.6; a small left surangular, IVPP V 23402.7; and a small right surangular, IVPP V 23402.8. IVPP V23403, consisting of a small right maxilla, IVPP V 23403.1; a small left dentary, IVPP V 23403.2; and a small left surangular, IVPP V 23403.3. IVPP V 23404, a larger left dentary. IVPP V 23405, consisting of a small left maxilla, IVPP V 23405.1 and a small left jugal, IVPP V 23405.2.

Locality and horizon: Locality 2, Jingangkou, Laiyang City, Shandong Province, China. Jingangkou Formation, Wangshi Group, Upper Cretaceous.

Diagnosis: Laiyangosaurus youngi is a saurolophine hadrosaurid distinguished by autapomorphies that include a prominent and narrow ridge on the lateral side of the nasal which forms the posterodorsal and posterior margin of the circumnasal depression, a primary ridge in most maxillary teeth slightly deflected posteriorly, a retroarticular process of the surangular that is dorsolateroposteriorly recurved, and an orbital margin that is wider than the infratemporal margin of the jugal. L. youngi can be further distinguished by a number of unique character combination that includes dorsal margin of nasal is flat, absence supracranial crest, a relatively shallow and rostrodorsally directed caudal margin of the jugal lacrimal process, and expended anteroposteriorly as well as one or more foramina on the rostral surface of the premaxilla. 


\section{DESCRIPTION}

\section{PREMAXILLA}

The small right premaxilla (IVPP V 23402.2) is incompletely preserved, missing the posterior portions of its lateral and dorsal processes (Fig. 2). The morphology of the oral margin of the premaxilla is rounded, although its anterior margin is slightly broken and the anterolateral corner is split. The oral margin of the premaxilla is lateroventrally deflected and dorsally expanded, forming a very thick 'lip-like' margin (Fig. 2a). In anterior view, the surface of the anterior margin of the premaxilla is rugose, and contains some vascular foramina (Fig. 2c), which are absent in most other members of Saurolophinae except Maiasaura (Prieto-Márquez et al. 2016). In ventral view, the oral margin of the premaxilla bears two layers of denticles, the anterior and posterior denticulate layers (Fig. 2b). There is no deep sulcus, but some foramina between these two layers. The anterior layer is broken, only the medial two denticles are preserved; these are blunt sub-conical and rostroventrally directed. However, the posterior denticles are pyramidal with a rostroventrally directed sharp apex, smaller than the anterior denticles. There are some vascular foramina between the two denticulate layers. Only the anterior segment of the lateral process of the premaxilla is preserved and is mediolaterally compressed. The dorsal process of the premaxilla is also mediolaterally compressed and triangular in lateral view, narrowing posteriorly to an apex; the lateral surface of the anterior portion of this process is concave medially and forms the medial wall of the anterior circumnarial depression. The ventral margin of the dorsal process forms the anterodorsal border of the posterior circumnarial depression, while the medial surface of this process is a flat articular face connected with the other side of premaxilla. In dorsal view, the vestibular promontory of the premaxilla expands anteroposteriorly and forms a fan-shape, as in Edmontosaurus annectens (Prieto-Márquez 2014, Mori et al. 2015). This vestibular promontory separates the anterior and posterior circumnarial depression, but the lateral circumnarial depression is covered.

NASAL

The small right nasal (IVPP V 23402.3) consists of a narrow rostrodorsal process and a wide caudal plate; both the rostral and caudal portions of this bone are slightly broken (Fig. 3). The rostrodorsal process is a mediolaterally compressed narrow stick that curves slightly ventrally, as in Kundurosaurus nagornyi (Godefroit et al. 2012), more curved than in Kerberosaurus manakini (Bolotsky and Godefroit 2004) and Edmontosaurus (Lambe 1917), and less curved in Gryposaurus (Gates and Scheetz 2014, Prieto-Márquez 2011). There is a narrow ridge at the lateral surface of the rostrodorsal process, extending ventrocaudally to the anterior margin of the caudal plate; in lateral view, this ridge forms the dorsal and caudal margin of the circumnarial depression (Fig. 3a). The caudal plate is mediolaterally compressed and slightly concaved laterally; its dorsoventral height is shorter than its anteroposterior length, although the caudal portion is missing. The medial surface of the caudal plate is flat, and while the posterior portion of the lateral surface bears a prefrontal facet, this facet is incompletely preserved, missing its posterior portion. There is a narrow groove extending anteroposteriorly at the ventral margin of the caudal plate that connects with the premaxilla.

\section{MAXILLA}

Four maxillae are included in this description (Fig. 4), a large left element (IVPP V 23401.1) (Fig. 4a, b, c), a small right element (IVPP V 23403.1) (Fig. 4h), and two small left elements (IVPP V 23405.1, IVPP V 23402.1) (Fig. 4d, e, f, g, i, j). In lateral view, the 
maxilla almost forms an isosceles triangle, with the dorsal process located centrally. Although either of these maxillae has a completely preserved anterior portion, IVPP V 23403.1 and IVPP V 23402.1 have almost complete anterodorsal and anteroventral

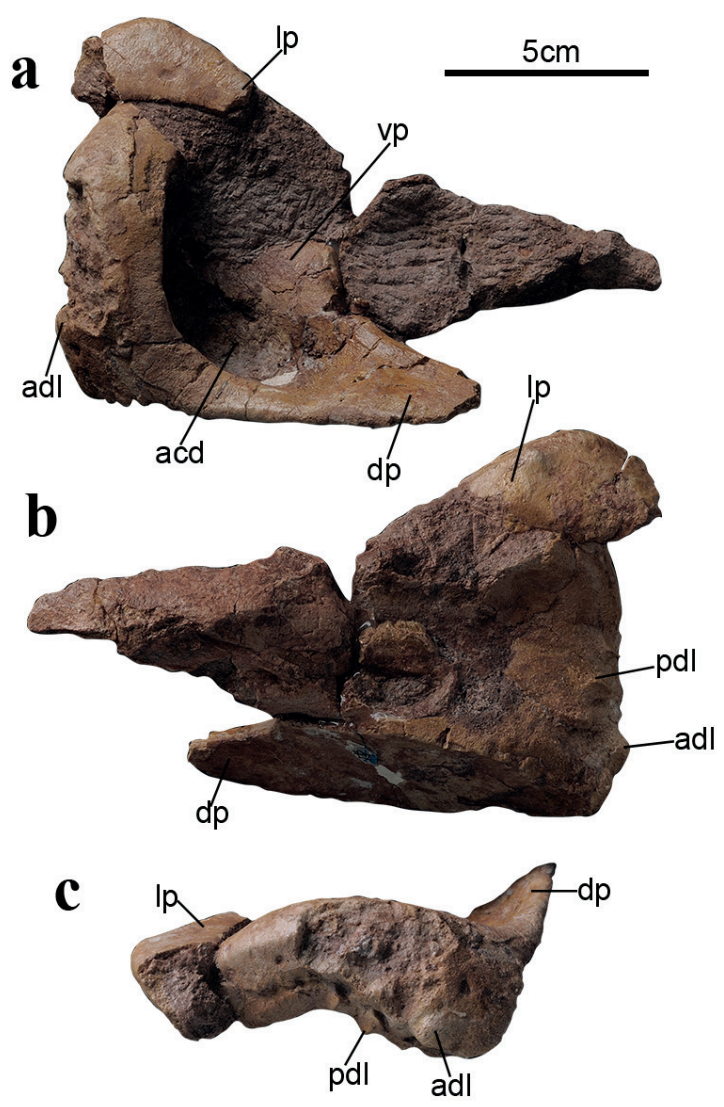

Figure 2 - Right premaxilla ( IVPP V 23402.2) of Laiyangosaurus. In dorsal (a), ventral (b), and anterior (c) views. Abbreviations: acd, anterior circumnarial depression; adl, anterior denticulate layer; dp, dorsal process; lp, lateral process; pcd, post circumnarial depression; vp, vestibular promontory. processes (Fig. 4h, i, j). Although the anterodorsal processes of IVPP V 23405.1 and IVPP V 23401.1 are almost absent, the marks of these can still be seen (Fig. 4b, d). The premaxillary facet is located between these processes, facing anterodorsally and extending caudodorsally, in front of the large maxilla foramen. The large oval maxilla foramen opens anterolateral, in the middle of anterodorsal margin of the maxilla, while the dorsal process of the maxilla forms a mediolaterally compressed isosceles triangle. The anterior margin of the dorsal process bears lacrimal facet, the lateral surface of this process is the jugal dorsal facet of maxilla (Fig. 5a). In lateral view, an anteroposteriorly extended groove lies in the ventral side of the dorsal process, and separates the jugal dorsal and ventral facet; this groove begins from the dorsal side of the large maxilla foramen, and ends to the dorsal side of dorsal jugal tubercle. The jugal ventral facet is an inverted triangle, articulating with the ventral half part of the anterior process of the jugal. The ventral margin of this facet forms a curved ridge, joined to the ectopterygoid ridge by the ventral jugal tubercle. There are also three or four small foramina in a row on the ventral side of the jugal ventral facet. While just in IVPP V 23402.1 is the palatine process completely preserved (Fig. $4 \mathrm{i}, \mathrm{j}$ ). Medioposteriorly to the dorsal process, the anteroposteriorly elongated palatine process forms the dorsomedial border of the maxilla, while in medial view, the anterior part of the palatine process slightly projects dorsally. In dorsal view, the

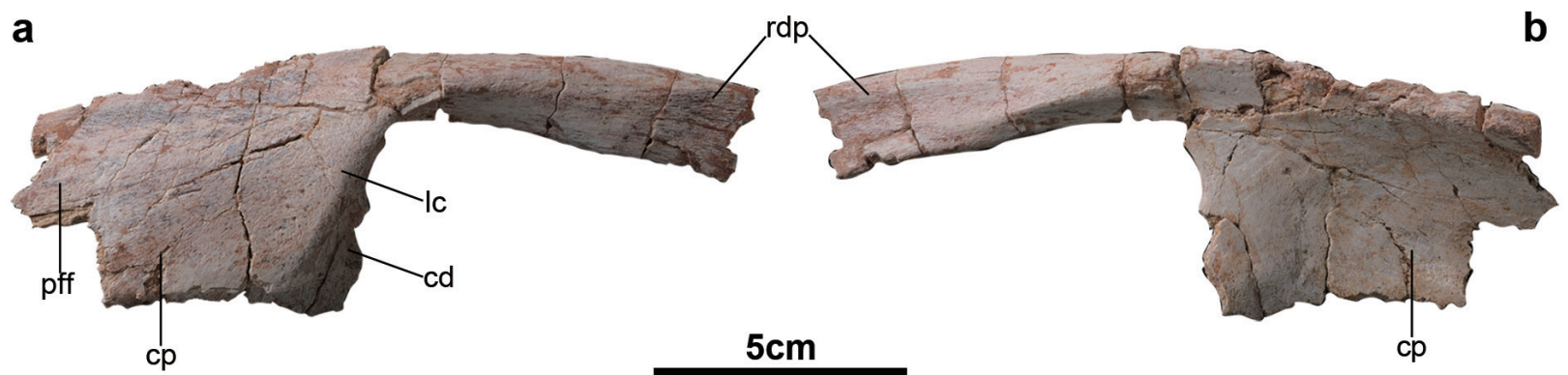

Figure 3 - Right nasal (IVPP V 23402.3) of Laiyangosaurus. In lateral (a), and medial (b) views. Abbreviations: cd, circumnarial depression; cp,caudal plate; lc, lateral crest; pff, prefrontal facet; rdp, rostrodorsal process. 

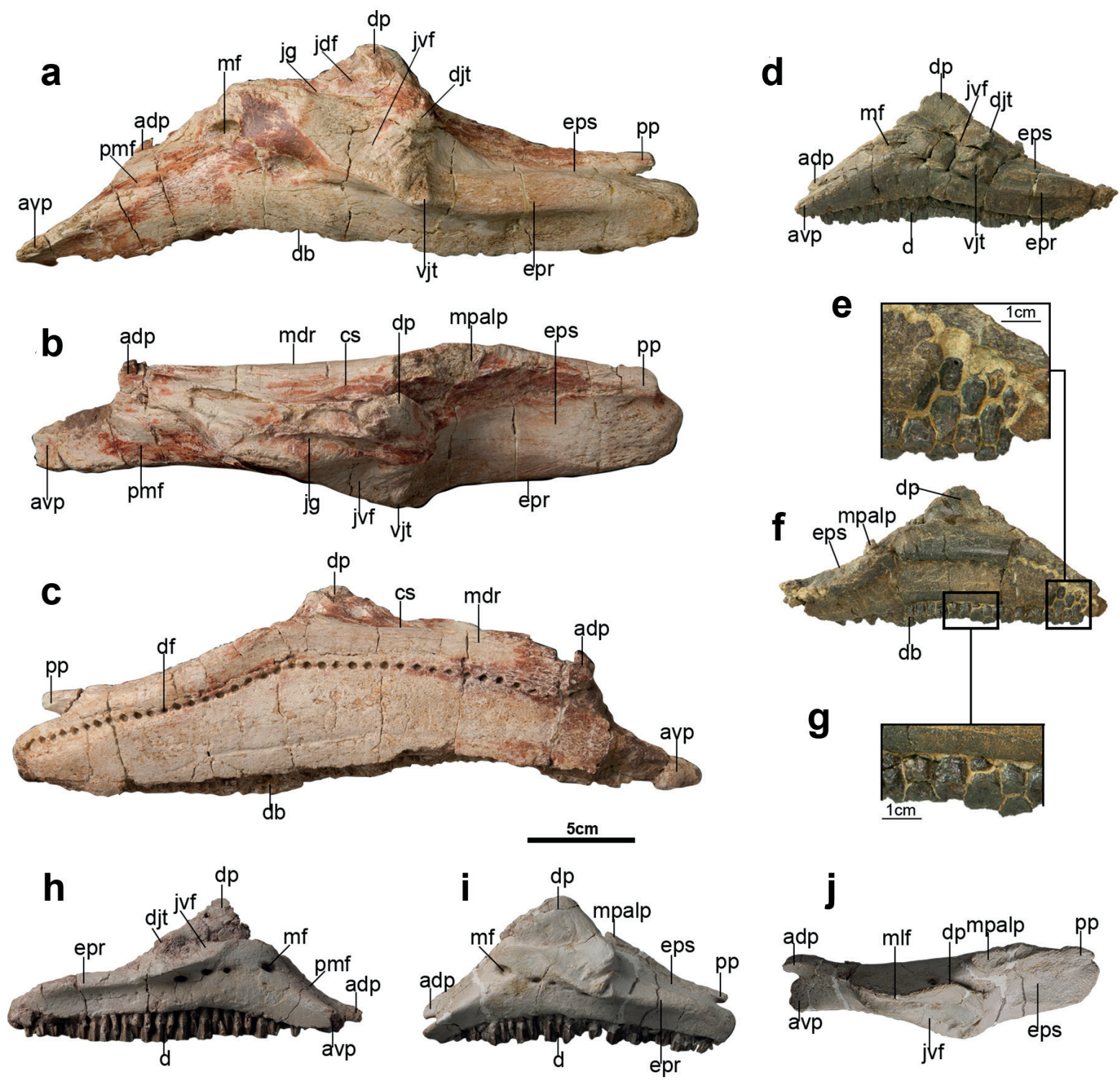

Figure 4 - Maxillae of Laiyangosaurus. a-c: left maxilla (IVPP V 23401.1) in lateral (a), dorsal (b), and medial (c) views; d-g: left maxilla (IVPP V 23405.1) in lateral view (d), medial view of maxillary teeth (e), medial view (f), medial view of occlusal surface of maxillary tooth (g); h: right maxilla (IVPP V 23403.1) in lateral view; i-j: left maxilla (IVPP V 23402.1) in lateral (i), and dorsal (j) views. Abbreviations: adp, anterodorsal process; avp, anteroventral process, cs, choanal shelf; db, dental battery; df, dental foramina; djt, dorsal jugal tubercle; dp, dorsal process; epr, ectopterygoid ridge; eps, ectopterygoid shelf; jdf, jugal dorsal facet; jg, jugal groove; jvf, jugal ventral facet; mdr, mediodorsal ridge; mf, maxillary foramen; mlf, maxilla lacrimal facet; mpalp, maxilla palatine process; pmf, premaxilla facet; pp, pterygoid process; vjt, ventral jugal tubercle. 
rectangular palatine facet is on the dorsal surface of the palatine process of the maxilla; between the palatine process and the dorsal jugal tubercle, a foramen connects with the maxilla foramen and the groove. The ectopterygoid ridge is located at the lateral surface of the ectopterygoid shelf; this ridge is dorsoventrally thick and horizontally oriented. The pterygoid process is missing in IVPP V 23405.1 and IVPP V 23403.1, but preserved in IVPP V 23401.1 and IVPP V 23402.1; this is a short dorsally extended process (Fig. 4c, i). The medial surface of the maxilla consists of a flat dental parapet, and curved row of alveolar foramina is located in the mid-height of the maxilla.

The largest of the preserved maxilla (IVPP V 23401.1) bears 41 tooth positions in the maxillary dental battery; the maxillary tooth density (the number of teeth per cm of maxillary dental battery) is 1.22 teeth $/ \mathrm{cm}$, while the number of alveolar positions of the other three small maxillae is between 33 and 35, and the maxillary tooth density is between 2.18 teeth/cm and 2.36 teeth $/ \mathrm{cm}$. These changes also all conform to hadrosaurid ontogeny rule, where the number of maxilla alveolar positions would relatively increase, and the maxillary tooth density would decrease (Prieto-Márquez 2008, 2014). In addition, because all these maxillae resemble each other in morphology, the small example must belong to a juvenile Laiyangosaurus, while the larger specimen represents an adult or a sub-adult individual.

Between one and two functional teeth are present on the occlusal plane (Fig. 4g) and more than three teeth of each type in the maxilla (Fig. $4 \mathrm{e})$. The maxillary tooth has a typical saurolophine morphology, diamond-shape in buccal view and with a single straight primary ridge on the buccal side (Prieto-Márquez 2008). Further, although the primary ridge is positioned caudally with respect to the midline of the crown, this is different from almost all the other hadrosauroids (PrietoMárquez 2008), with the exception of the basal taxa Bactrosaurus johnsoni (Gilmore 1933) and Ouranosaurus nigeriensis (Taquet 1976). There is no small denticle present on the anterior and posterior margin of the maxillary tooth.

JUGAL

The small left jugal (IVPP V 23405.2) is almost completely preserved; just the postorbital process and quadratojugal flange have some fractures (Fig. $5 \mathrm{~b}, \mathrm{c})$. This element was found near the maxilla (IVPP V 23405.1); the articular facets of these two bones merge perfectly, and therefore they likely belong to the same individual (Fig. 5a). In lateral view, the surface is flat and the shape of the rostral process forms an asymmetrical triangle, the anterior spur of the rostral process is wedge-shaped that is located at the dorsal part of the rostral process. The ventral margin of the anterior spur is horizontally oriented, the posterior segment of the ventral margin of the rostral process is slightly broken. The dorsal margin of the rostral process is laterally deflected and postodorsally extended, bears a narrow and shallow groove on the lacrimal facet of the jugal. The dorsal apex of the rostral process encapsulates the lacrimal process of jugal in lateral view. The relatively shallow caudal margin of the lacrimal process of the jugal is rostrodorsally directed, and forms little of the rostroventral margin of the orbital rim. However, this caudal margin of the lacrimal process of the jugal is deep and posterodorsally deflected in other members of Saurolophinae (Prieto-Márquez 2008, 2010b). In the medial view, there is also a wide maxillary facet on the jugal in the medial surface of the rostral process which incorporates a dorsoventrally expanded ridge, limiting the posterior margin of the maxilla facet. The dorsal segment of this ridge is anteriorly deflected and touches the lacrimal facet on the rostral process which also bears a narrow and shallow palatine process of the jugal in medial view. In addition, an anteroposteriorly 


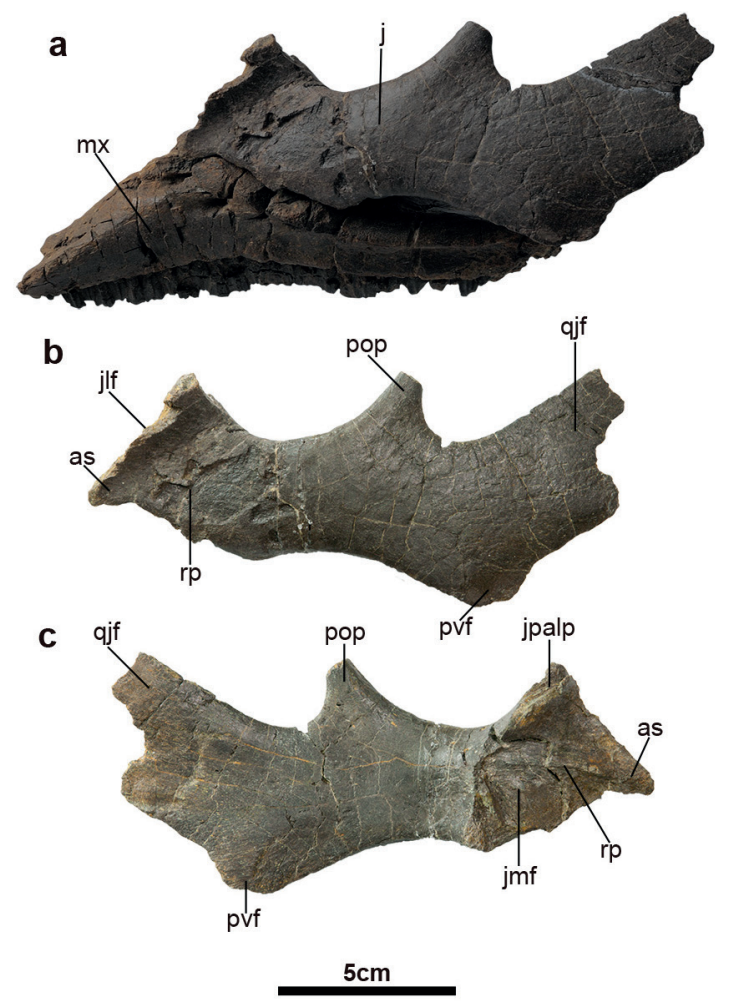

Figure 5 - Jugal of Laiyangosaurus. a: the combination of the left maxilla (IVPP V 23405.1) and left jugal (IVPP V 23405.2) in lateral view; b-c: left jugal (IVPP V 23405.2) in 'lateral (b) and medial (c) views. Abbreviations: as, anterior spur; j, jugal; jmf, jugal maxilla facet; jpalp, jugal palatine process; mx, maxilla; pop, postorbital process; pvf, posteroventral flange; qjf, quadratojugal flange; rp, rostral process.

expanded ridge separates the maxillary facet into dorsal and ventral parts. The ventral margin of the orbit is wider than that of the infratemporal fenestra, while the orbital constriction is slightly lower than its infratemporal counterpart. There is a wide and slightly dorsally convex ventral margin of the jugal between the rostral process and the caudoventral flange; the posteroventral flange of the jugal is only moderately expanded and the ratio between the maximum dorsoventral depth of the posteroventral flange and the minimum depth of the infratemporal constriction of the jugal is 1.2. At the same time, the postorbital process is posteriorly deflected, although its dorsal segment is missing; the quadratojugal flange is incompletely preserved, but it still has an auricular shape. The anterodorsal and posteroventral margins of the quadratojugal flange are sub-parallel, and there is a relatively shallow concavity between the posteroventral and quadratojugal flanges; the quadratojugal facet is located at the dorsal portion of its quadratojugal flange.

\section{SQUAMOSAL}

Two squamosals have been unearthed from this site (Fig. 6). Of these, the larger right element (IVPP V 23401.2) is broken, and the most of the postorbital process and postquadratic process is missing. The small left squamosal (IVPP V 23402.4) is nearly complete, with just a little abrasion on its anterior and posterior portions. In lateral view, the triangle postorbital process of the squamosal is mediolaterally compressed, while the lateral surface of this process bears a wide and shallow concave postorbital articular facet to receive the squamosal process of the postorbital. In lateral view, the posterior segment of the postorbital facet forms a rectangle (Fig. 6a, c), while in IVPP V 23401.2, the posterior margin of the postorbital facet is located above anterior portion of the quadrate cotyle (Fig. $6 a)$, ending above the prequadratic process in IVPP V 23402.4 (Fig. 6c). This triangular prequadratic process is anteroventrally extended, a little longer than the anteroposterior length of the quadrate cotyle. The postquadratic processes are both broken on these two squamosals; their preserved portions show that the postquadratic process is robust and ventrally extended. The quadrate cotylus is located between the pre- and postquadratic processes to receive the quadrate head, while the posterior portion and the parietal process are both missing in medial view (Fig. 6b, d). 

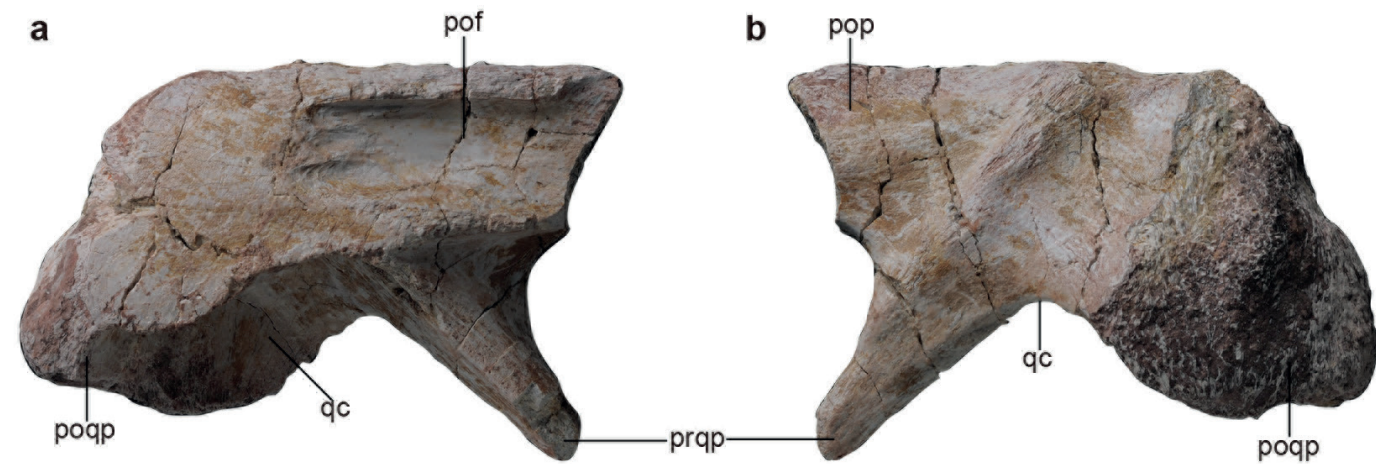

c

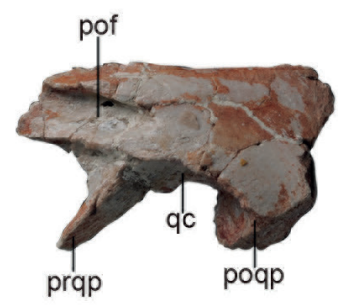

d

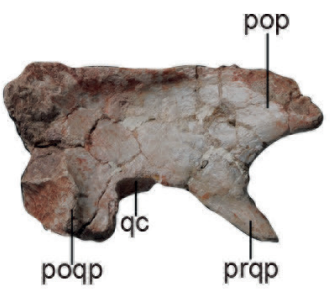

$5 \mathrm{~cm}$

Figure 6 - Squamosals of Laiyangosaurus. a-b: right squamosal (IVPP V 23401.2) in lateral (a) and medial (b) views; c-d: left squamosal (V23402.4) in lateral (c) and medial (d) views. Abbreviations: pof, postorbital facet; pop, postorbital process; poqp, postquadratic process; prqp, prequadratic process; qc, quadrate cotylus.
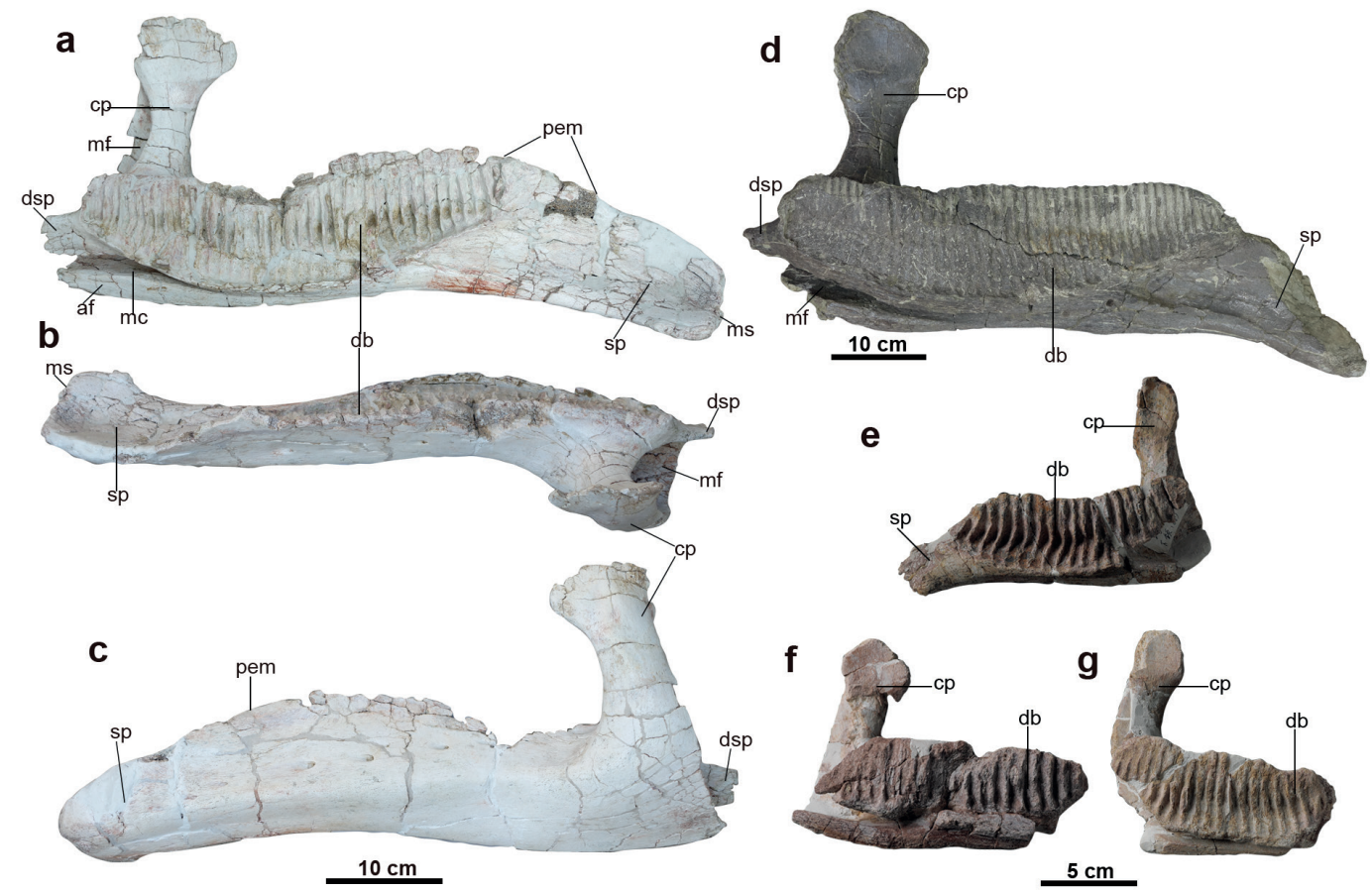

Figure 7 - Dentaries of Laiyangosaurus. a-c: left dentary (IVPP V 23401.3) in medial (a), dorsal (b), and lateral (c) views; d: left dentary (IVPP V 23404) in medial view; e: right dentary (IVPP V 23402.5) in medial view; f-g: left dentaries (IVPP V 23402.6, V 23403.2) in medial view. Abbreviations: af, angular facet; cp, coronoid process; db, dental battery; dsp, dentary splenial process; mc, mackelian canal; mf, mackelian fossa; ms, mandibular symphysis; pem, proximal edentulous margin; sp, symphysis process. 


\section{DENTARY}

Two large (IVPP V 23401.3, IVPP V 23404) and three small dentaries (IVPP V 23402.5, IVPP V 23402.6, IVPP V 23403.2) are referred to this taxon (Fig. 7). Of these, the large examples are both right elements and IVPP V 23401.3 is nearly completely preserved (Fig. 7a-c), while the symphysis process of IVPP V 23404 is missing (Fig. 7d). In contrast, IVPP V 23402.5 is a small right dentary that is broken in anterior and posterior portions, and the other small left dentaries (IVPP V 23402.6, V 23403.2) are both badly preserved, lacking their anterior half portions (Fig. 7e-g). All of these specimens are mediolaterally compressed and laterally concaved, while the anterior segment of each bears an edentate symphysis process. All the symphysis processes of these dentaries exhibit different degrees of breakage; in the largest specimen, IVPP V 23401.3, the symphysis process is relatively complete and slightly medioventrally deflected, extending nearly half the length of the dental battery. The dorsal margin of IVPP V 23401.3 is also broken, so that the extent of the proximal edentulous margin cannot be determined. In IVPP V 23404, the dorsal border of the battery is straight and nearly parallel with the ventral border, while the posterior part of the ventral border is convex. No tooth is preserved in the battery of any of these dentaries; 39 narrow alveoli are preserved in the battery of IVPP V 23401.3, while 46 are present in IVPP V 23404, and 21 are seen in IVPP $\mathrm{V}$ 23402.5. The anterior segment of this battery is broken in IVPP V 23402.6 and IVPP V 23403.2; the density of teeth is around 1 tooth $/ \mathrm{cm}$ in the larger specimens (IVPP V 23401.3, V 23404), rising to 1.6 teeth $/ \mathrm{cm}$ in the smaller specimens (IVPP V 23402.5). This situation is similar to observed variation in maxillary tooth density that is seen during hadrosaurid ontogeny (Prieto-Márquez 2008, 2010b). The posterior end is greatly caudally extended beyond the caudal border of the coronoid process in the larger specimens (IVPP V 23401.3, IVPP V 23404), while the posterior portion of three small dentaries are all broken. A triangular splenial process is also present, posteriorly connected with the battery in each specimen, and caudally tapered, while a large meckelian fossa is seen between the splenial and coronoid processes, connected with the narrow meckelian canal which lies ventrally to the posterior part of the dental battery. The coronoid process in each specimen is also slightly anteriorly inclined, with an apex that is mediolaterally compressed. The lateral side of the apex is convex, and the medial side is slightly concave. There is a anteroposteriorly expanded ridge at the dorsal part of the lateral surface of the dentary, containing a series of foramina on the dorsal side of the ridge.

\section{SURANGULAR}

Three small surangulars are referred to this taxon (Fig. 8), including two left elements (IVPP V 23402.7, IVPP V 23403.3) and a right (IVPP V 23402.8). Of these, specimen IVPP V 23402.7 is nearly complete, while the other two lack their anterior and posterior portions. A triangular concavity is present on the mediodorsal surface of the anterior part of the surangular; the lateral surface of this dentary facet is not obvious and just a weak profile can be seen. No foramina are present on the lateral surface of the surangular; the anterior portion of this element has a dorsally extended ascending process. This mediolaterally compressed ascending process is thin and strap-like in dorsal view, and narrows dorsally in lateral view. In the centre of dorsal side of the surangular, a tall medial sagittal ridge is also present, dorsoventrally compressed, and extended from the middle of this element as far as the posterior articular process. On the dorsal side of the medial sagittal ridge, a D-shaped lateral lip is also present that is dorsoventrally thick. In medial view, a shallow depression is located posteriorly to this lip, connected with the glenoid 


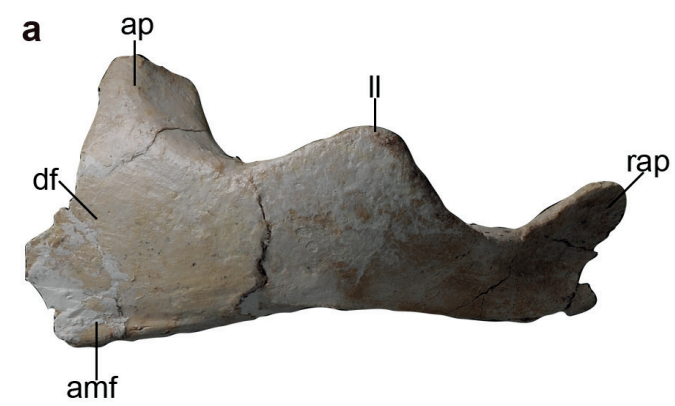

d
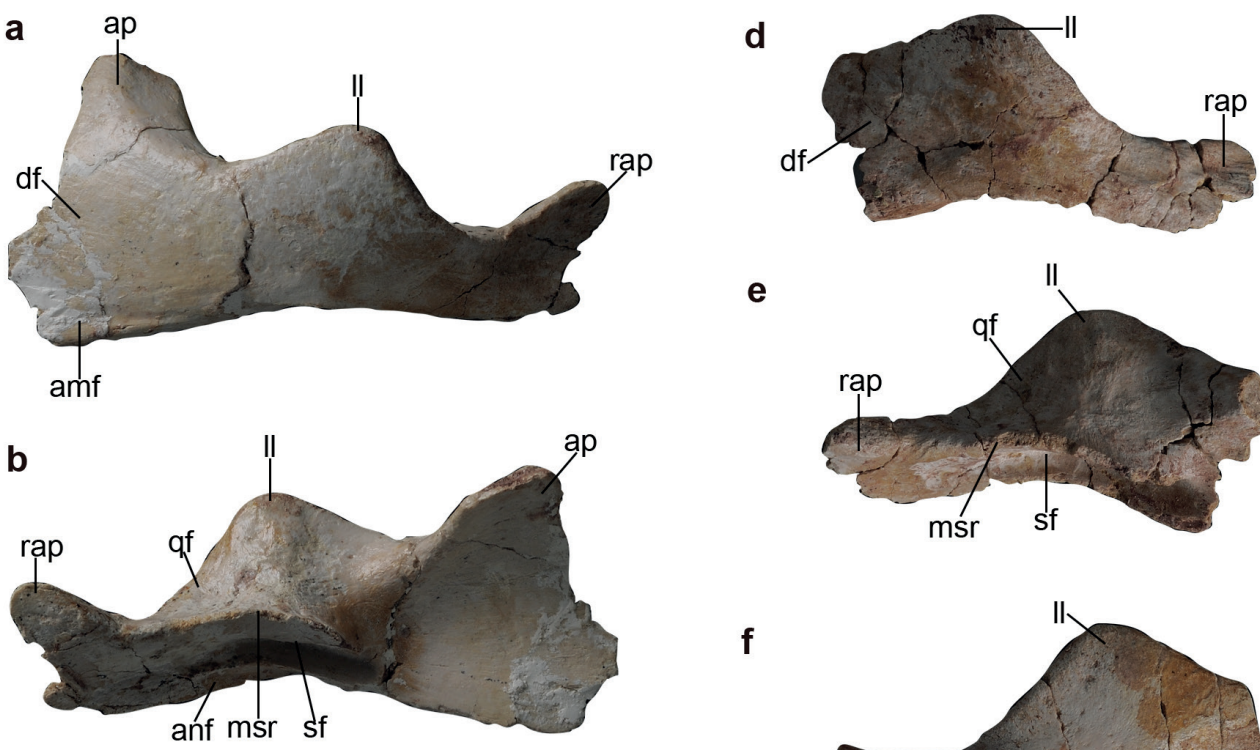

e

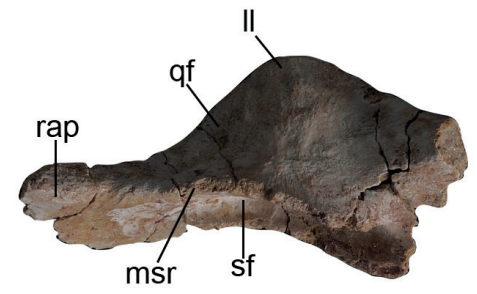

$\mathbf{f}$
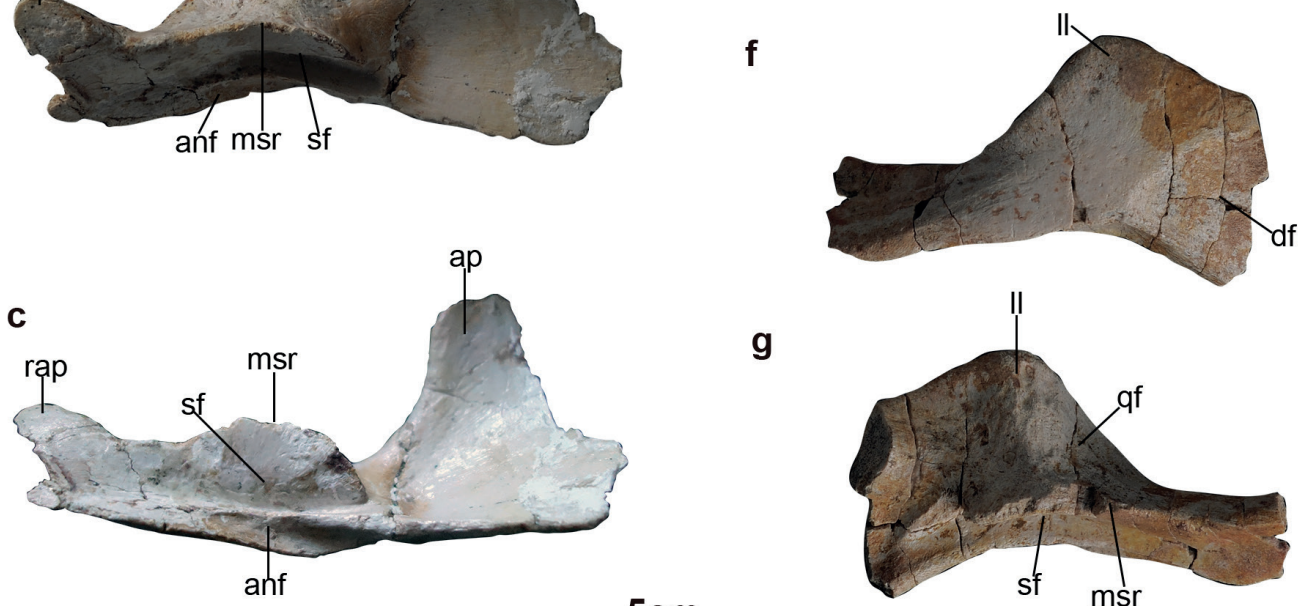

$5 \mathrm{~cm}$

g

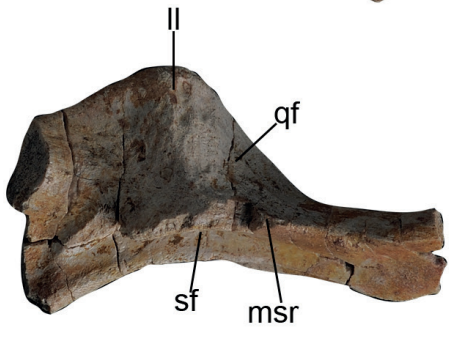

Figure 8 - Surangulars of Laiyangosaurus. a-c: left surangular (IVPP V 23402.7) in lateral (a), medial (b), and vental (c) views; d-e: left surangular (IVPP V 23403.3) in lateral (d) and medial (e) views; f-g: right surangular (IVPP V 23402.8) in lateral (f) and medial (g) views. Abbreviations: amf, anteromedial flange; anf, angular facet; ap, ascending process; df, dentary facet; 11, lateral lip; msr, medial sagittal ridge; qf, quadrate facet; rap, retroarticular process; sf, spienial facet.

facet for the quadrate, while the ventral side of the medial sagittal ridge is an articular surface for the splenial. In addition, another shallow sagittal ridge is also present, parallel with the medial sagittal ridge, below the splenial facet. The angular facet is located in the ventral side of this ridge and forms the ventral margin of the surangular. The posterior portion of the surangular bears a mediolaterally compressed retroarticular process, which is deflected dorsolateroposteriorly. This retroarticular process in the other members of the Saurolophinae is deflected posteriorly (Prieto-Márquez 2008, 2010b).

\section{COMPARISON}

Laiyangosaurus exhibits a number of characteristics of Hadrosauroidea, including the presence of a premaxilla with a transversely expanded and edentulous margin and a ventral deflection of the dentary that occurs rostrally (Norman 2004, PrietoMárquez 2010b, Xing et al. 2014a). As discussed, three genera of hadrosauroids have been reported 
from Laiyang, Tanius (Wiman 1929), Tsintaosaurus (Young 1958), and Shantungosaurus (Hu 1973). In recent phylogenetic analyses, Tanius was assigned to the non-hadrosaurid hadrosauroids, whereas Tsintaosaurus and Shantungosaurus are assigned to Hadrosauridae, Tsintaosaurus is a member of Lambeosaurinae, and Shantungosaurus is a saurolophine dinosaur (Prieto-Márquez 2010b, Xing et al. 2014a).

Three species have been referred to the genus Tanius, of which the type species, T. sinensis, includes a partial skull (Wiman 1929), while the other two species, T. chingkankouensis and $T$. laiyangensis, are known only from postcranial skeletons (Young 1958, Zhen 1976) and do not overlap with Laiyangosaurus. In addition, because the premaxilla, maxilla, dentary, and surangular of $T$. sinensis are not preserved, only the jugal and squamosal can be used to distinguished this material from Laiyangosaurus. The jugal and squamosal of $T$. sinensis display characteristic basal hadrosauroid morphology (Prieto-Márquez 2008, $2010 \mathrm{~b}$ ); the rostral process of the jugal is relatively shallow dorsoventrally, the posterodorsal border of the rostral process bears a remarkable horizontally oriented ridge and continues to the dorsal border of the rostral process, making a deep concave maxilla facet in the medial surface of the rostral process. At the same time, the rostral process of the jugal of Laiyangosaurus is deeper dorsoventrally; the dorsal border of the medial surface of this process comprises a dorsoventrally expanded ridge, while the dorsal segment of this ridge is anteriorly deflected and the maxillary facet is shallow. The squamosal of $T$. sinensis is relatively deeper dorsoventrally than in Laiyangosaurus, while the premaxilla, maxilla, and dentary of this new taxon also exhibit a series of Hadrosauridae characteristics, including the fact that the oral margin of the premaxilla is strongly deflected ventrally, the jugal facet of the maxilla is subtriangular and connects with the ectopterygoid ridge, a large maxillary foramen is present on the dorsal surface of the rostrodorsal margin of the rostral part of the maxilla, less than six maxillary foramina form a row oriented rostrodorsally below the jugal articulation, the coronoid process of the dentary is anteriorly deflected, the number of dental teeth is more than 30 , the caudal end of the dental battery is located caudal to the caudal margin of the coronoid process. The surangular lacks a surangular foramen and accessory foramen (Horner et al. 2004, Prieto-Márquez 2008, 2010b).

Tsintaosaurus also has a premaxilla, maxilla, nasal, squamosal, dentary, and surangular which can be compared with Laiyangosaurus. While this latter taxon has some typical saurolophine features, there are nevertheless some significant differences with Tsintaosaurus, a representative of Lambeosaurinae (Horner et al. 2004, Prieto-Márquez 2008, 2010b). The maxilla of Laiyangosaurus bears an anterodorsal process, absent in Tsintaosaurus, and the anteroventral process of Laiyangosaurus is not deflected ventrally like in Tsintaosaurus. The dorsal process of the maxilla in Laiyangosaurus is also a flat isosceles triangle, and its apex is located in the middle of maxilla anteroposteriorly; this process is dorsoventrally taller than its wide with a peaked and caudally inclined apex in Tsintaosaurus. The rostral edentulous region of the dentary is slightly deflected ventrally in Laiyangosaurus, while this is strongly deflected in Tsintaosaurus. In addition, Laiyangosaurus has a wide and shallow ventral margin of the jugal, unlike lambeosaurines which have a jugal ventral margin that has a relatively narrow and deep embayment (Prieto-Márquez 2008, 2010b).

Shantungosaurus was regarded by $\mathrm{Hu}$ (1973) as a member of Saurolophinae. Although most of the fossil material referred to this genus comes from the Upper Cretaceous Wangshi Group of Zhucheng (Fig. 1), some elements were also found in Laiyang (Hu et al. 2001). Shantungosaurus includes most of the skull, and is the largest known ornithischian dinosaur. Comparisons show 
that Laiyangosaurus and Shantungosaurus share some important features, including the fact that the dorsal border of the nasal is flat and lacks a crest, the dorsal part of oral margin of premaxilla is expanded posterodorsally and forms a thick 'liplike' margin, the outline of maxilla is triangular, and the apex of the dorsal process of this element is in the middle of the maxilla. However, there are also some significant differences; although the rostral process of the nasal in Laiyangosaurus is generally flat, it is nevertheless more downwardly curved than in Shantungosaurus. The lateral surface of the nasal in Laiyangosaurus also bears a narrow crest that runs from the rostral process to the anterior part of caudal plate, while this crest is flat in Shantungosaurus (Xing et al. 2014b). The maxilla of Shantungosaurus is distinctly larger than Laiyangosaurus, and the latter also has a very shallow and anteroposteriorly expanded palatine process of the maxilla; this process is deeper and hook-like in Shantungosaurus (Hu et al. 2001). During hadrosaurid ontogeny, the skull becomes relatively more elongate anteroposteriorly, implying that a similar pattern took place in maxillary development (Mori et al. 2015). The height (the distant from the apex of the dorsal process to the margin of tooth battery) to length (the distant from the anterior apex of the anteroventral process to the end of the ectopterygoid shelf) ratio of the maxilla is also reduced as the animal grows (Mori et al. 2015). Data show that the ratio of the big maxilla of Laiyangosaurus (IVPP V 23401.1) is 0.29 , while the ratio of other three small maxillae (IVPP V 23405.1, IVPP V 23403.1, IVPP V 23402.1) fall between 0.35 and 0.38 , indicative of ontogeny. At the same time, this ratio is 0.36 in Shantungosaurus, larger than the biggest specimen of Laiyangosaurus and equal to juveniles. Thus, the ratio of sub-adult and juvenile Shantungosaurus, the same size as Laiyangosaurus, would be more than 0.36. Compared with similarly-sized material of Shantungosaurus, Laiyangosaurus also has a relatively lower and longer maxilla. The former has 55-57 maxillary teeth, more than Laiyangosaurus (33-41 teeth), and the density of maxillary teeth in Shantungosaurus $(0.89-0.96$ teeth/cm) is less than that in Laiyangosaurus (1.20-2.53 teeth/cm).

An additional four genera of Saurolophinae have been described from Asia, including Wulagasaurus (Godefroit et al. 2008), Kerberosaurus (Bolotsky and Godefroit 2004) and Kundurosaurus (Godefroit et al. 2012) from the Heilongjiang (Amur) River area between China and Russia, and Saurolophus angustirostris (Rozhdestvensky 1952) from Mongolia.

Of these Asian taxa, Wulagasaurus was discovered in the Yuliangze Formation within the Upper Cretaceous of the Wulaga region, Jiayin, in Heilongjiang Province, China (Godefroit et al. 2008). This taxon consists of a partial skull, and so its maxilla, jugal, and dentary can be compared with Laiyangosaurus. The jugal facet of the maxilla is very concave in Wulagasaurus, while this facet in Laiyangosaurus is only slightly concave. In addition, the posterior portion of the ectopterygoid ridge of the maxilla is deflected ventrally in Wulagasaurus, while this entire region is nearly horizontal in Laiyangosaurus. The jugal of Wulagasaurus is relatively elongate, and the apex of the rostral process is located at the level of its dorsalventral midpoint (Godefroit et al. 2008, Xing et al. 2012). However, this element of Laiyangosaurus is relatively short as well as the apex is located at dorsal part of the rostral process. In addition, the dentary of Wulagasaurus are also obviously slender than Laiyangosaurus (Godefroit et al. 2008, Xing et al. 2012).

Kerberosaurus consists of some disarticulated skull bones that were collected from the Udurchukan Formation within the Upper Cretaceous at Blagoveschensk in the Amur Region of the Russian Far East (Bolotsky and Godefroit 2004). In comparison, Kerberosaurus possesses a lower maxilla with a wider dorsal process than 
Laiyangosaurus, as well as a deep and hook-like palatine process. This process is lower and extended anteroposteriorly in Laiyangosaurus. At the same time, Kerberosaurus has a round rostral process of jugal, which is triangular in Laiyangosaurus. The lateral side of nasal of Kerberosaurus bears a very prominent, flattened crest, which is narrow in Laiyangosaurus. Finally, the rostral process of the nasal is more downwardly deflected in Laiyangosaurus compared to Kerberosaurus (Bolotsky and Godefroit 2004).

Kundurosaurus was found in the Udurchukan Formation within the Upper Cretaceous at Kundur in the Amur Region of the Russian Far East (Godefroit et al. 2012). The material of Kundurosaurus includes a number of disarticulated skull and postcranial bones, and this taxon shares several important features with Laiyangosaurus. For example, the absence of a hook-like palatine process of the maxilla, the rostral process of jugal has an asymmetric spur, and the rostral process of the nasal slightly deflected downwards. At the same time, there are also several significant differences; although most of the postorbital process of the jugal is not preserved in Laiyangosaurus, its basal part indicates posterodorsal extension while this process in Kundurosaurus ascends at a nearly $90^{\circ}$ angle. The lateral crest of the nasal is also flatter in Kundurosaurus than in Laiyangosaurus, and the dentary is slender (Godefroit et al. 2012).

Saurolophus angustirostris comes from the Nemegt Formation, Nemegt, Mongolia (Rozhdestvensky 1952). The dorsal part of the oral margin of the premaxilla in this taxon is slightly deflected and extended posterodorsally, without the thick 'lip-like' oral margin in Laiyangosaurus. The maxilla and lacrimal contact one another anterior to the rostral process of the jugal in S. angustirostris, while the rostral process of this element separates the maxilla and lacrimal in Laiyangosaurus. The nasal of $S$. angustirostris is elongate and extends posterodorsally over the cranium, forming a solid rod-like crest. This crest is present in juvenile individuals, and become enlarged during ontogeny (Bell 2011, McGarrity et al. 2013). Although the small nasal of Laiyangosaurus, IVPP V 23402.3, would belong to a juvenile individual, the flat nasal without crest reflects a true morphological characteristic.

Compared with other members of the Saurolophinae, Laiyangosaurus is unique. Another species of Saurolophus, S. osborni, was found in the Horseshoe Canyon Formation, Alberta, Canada (Brown 1912), while Prosaurolophus comprises two species, P. maximus (Brown 1916) and $P$. blackfeetensis (Horner 1992) from the Campanian (Upper Cretaceous) of North America. S. osborni and Prosaurolophus share some features with $S$. angustirostris (Prieto-Márquez 2008, 2010b), distinct from Laiyangosaurus.

Edmontosaurus comprises three species, E. regalis (Lambe 1917), E. annectens (Marsh 1892) and E. saskatchewanensis (Sternberg 1926) from the Maastrichtian (Upper Cretaceous) of North America. Edmontosaurus has a flat dorsal margin of nasal, as in Shantungosaurus and Kerberosaurus, unlike Laiyangosaurus and Kundurosaurus which possess a slightly downward curved nasal (Godefroit et al. 2012, Xing et al. 2014b). The anterodorsal process of the maxilla is more elongate, while the rostral process is dorsoventrally deeper in Edmontosaurus than in Laiyangosaurus (Prieto-Márquez 2008, 2010b, 2014). Laiyangosaurus has a prominent and narrow ridge on the lateral side of the nasal that borders posterodorsally and posteriorly the circumnasal depression, while the other flat-headed saurolophines, Edmontosaurus, Shantungosaurus, Kerberosaurus, and Kundurosaurus, also have a wide and flat ridge (Godefroit et al. 2012, Xing et al. 2014b). Ugrunaaluk (Mori et al. 2015) is a new flat-headed hadrosaurid from the Price Creek Formation, northern Alaska, U.S.A. The nasal of size class 1 Ugrunaaluk and the small nasal 
of Laiyangosaurus are equal in size, while this element in the former also has a wide and flat lateral ridge (Mori et al. 2015). Consequently, the narrow lateral crest of nasal in Laiyangosaurus cannot be ontogenetic. In addition, the posterodorsal and posterior magin of circumnasal depression in the solid-crest saurolophines is distinctly different with the narrow ridge of Laiyangosaurus. As a result, this feature, the presence of a narrow ridge on the lateral side of the nasal, can be considered an autapomorphy of Laiyangosaurus.

Kritosaurus was discovered in the Upper Kirtland Formation, San Juan County, New Mexico, U.S.A (Brown 1910). This taxon comprises several partial skulls, and has a deep ventral process of the rostral process of the jugal (Prieto-Márquez 2014), while there is no prominent process in the caudoventral margin of the rostral process in Laiyangosaurus. There is also a relatively narrow and deep embayment at the ventral margin of the jugal, between the caudoventral flange and the rostral process in Kritosaurus (Prieto-Márquez 2014); in Laiyangosaurus, this is a relatively wide and shallow embayment.

Gryposaurus comprises three species, $G$. notabilis (Lambe 1914), G. latidens (Horner 1992), and G. monumentensis (Gates and Sampson 2007) from the Campanian of North America. The oral margin of the premaxilla of Gryposaurus is thin, without a 'lip-like' structure, while the maxilla and lacrimal of Gryposaurus contact one another, as in Saurolophus and Prosaurolophus, but distinct from Laiyangosaurus (Gates and Sampson 2007, Prieto-Márquez 2008, 2010b, 2011). Gryposaurus has a deep ventral process of the rostral process of the jugal, which resembling Kritosaurus (PrietoMárquez 2008, 2010b, 2011, 2014). The nasal of Gryposaurus is arched anterodorsally, forming a 'hook-like' crest (Gates and Sampson 2007).

Brachylophosaurini (Gates et al. 2011) is a clade of saurolophine dinosaurs from the Campanian of North America that includes Acristavus (Gates et al. 2011), Brachylophosaurus (Sternberg 1953), Maiasaura (Horner and Makela 1979), and Probrachylophosaurus (Fowler and Horner 2015). Members of this clade all have a nasal crest or an arched hook-nose. They share some important features, as distinct from Laiyangosaurus (PrietoMárquez 2008, 2010b, Fowler and Horner 2015). The lateral corner of oral margin of premaxilla bears a process in Brachylophosaurini, while this corner is round in Laiyangosaurus. In addition, the rostral process of the jugal in Brachylophosaurini is nearly symmetric dorsoventrally, while the rostral process of this element in Laiyangosaurus bears an asymmetric wedge-shaped anterior spur.

Two genera of saurolophine dinosaurs have been reported from South America, Secernosaurus (Brett-Surman 1979) from the San Jorge Formation, Upper Cretaceous, Rio Chico, Patagonia, Argentina, and Willinakaqe (Juárez Valieri et al. 2010) from the Allen Formation, Upper Cretaceous, Salitral Moreno, Río Negro Province, southern Argentina. They both have partial skull bones, and their symphyseal of dentary is more strongly deflected medially than that in Laiyangosaurus (PrietoMárquez and Salinas 2010, Juárez Valieri et al. 2010). Additionally, the dentary of Willinakaqe is relatively more robust and shorter than that in Laiyangosaurus, and the coronoid process is subvertical, unlike the anteriorly inclined coronoid process in Laiyangosaurus (Juárez Valieri et al. 2010). The ectopterygoid ridge of maxilla of Secernosaurus is obviously inclined ventrally, while the ectopterygoid ridge is nearly paralleled with the tooth row (Prieto-Márquez and Salinas 2010).

\section{PHYLOGENETIC ANALYSIS}

We conducted a maximum parsimony analysis to assess the phylogenetic position of Laiyangosaurus youngi within hadrosauroid iguanodontians. This analysis is based on the 273-character matrix of 
Prieto-Márquez (2016), consisting of 189 cranial and 84 postcranial equally weighted characters (see Appendix A). Including L. youngi, the taxonomic sample consisted of 62 hadrosauroid species (including 16 outgroups to Hadrosauridae, 23 saurolophines, and 21 lambeosaurines). The optimal tree(s) search was conducted in TNT version 1.1 (Goloboff et al. 2008). A heuristic search of 10,000 replicates using random addition sequences was performed, followed by branch swapping by tree bisection-reconnection holding ten trees per replicate.

The analysis resulted in four most parsimonious trees of 1,000 steps each (Consistency Index D 0.398, Retention Index D 0.780); the best score was found in 8,538 of the 10,000 replicates. The strict consensus tree shows that L. youngi is in Saurolophinae, which is in consistent with the result of our anatomical comparison (Fig. 9). This analysis also shows that L. youngi lies in a monophyletic

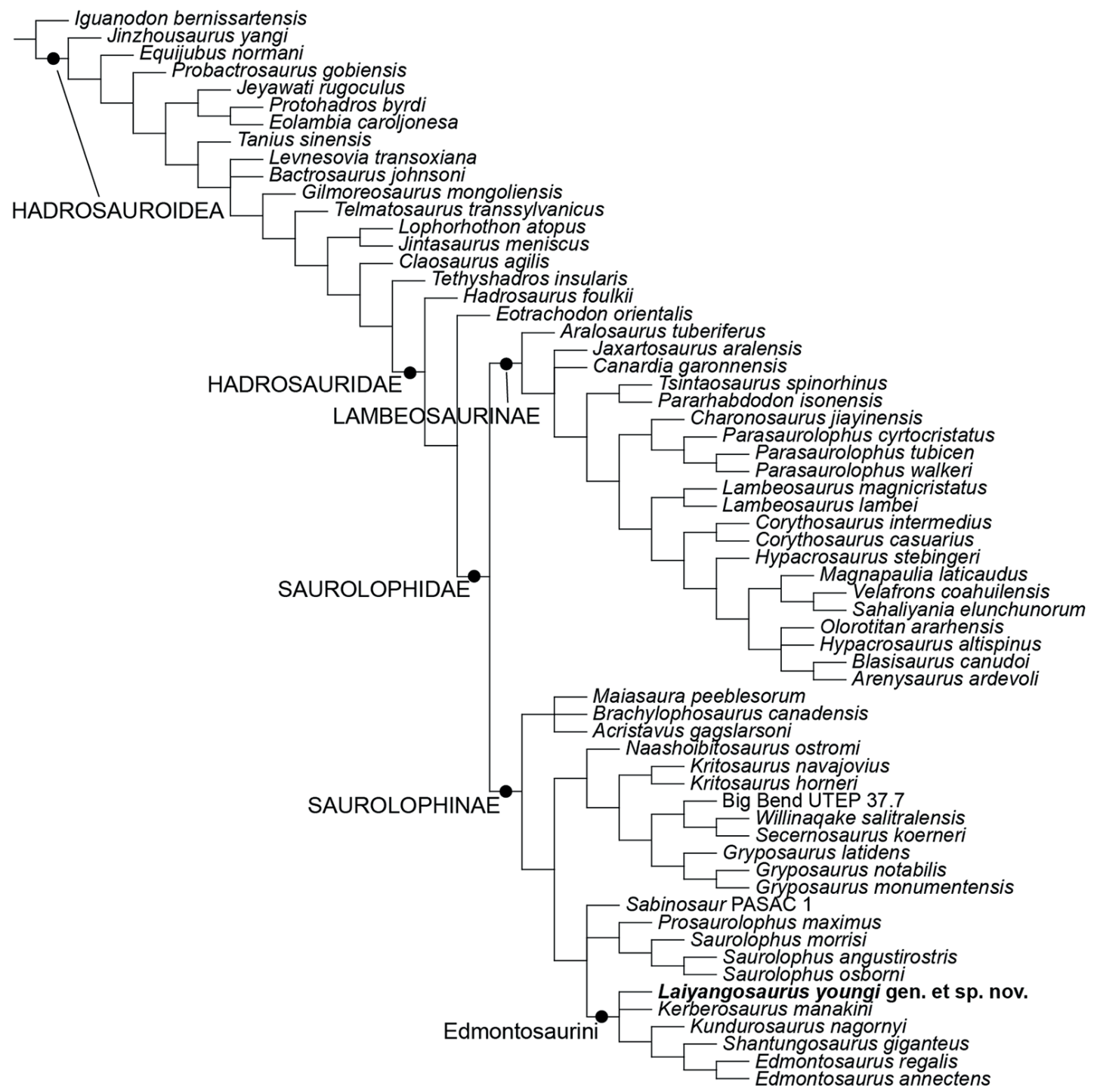

Figure 9 - Strict consensus tree of the four most parsimonious trees resulting from the phylogenetic analysis of hadrosauroid relationships, showing the position of Laiyangosaurus youngi. 
group, which is known as Edmontosaurini, supported by four synapomorphies: present a ridge bordering the caudodorsal margin of the circumnarial depression (character $75[1,2]$ ), maxilla-lacrimal contact largely covered externally by the jugal-premaxilla contact (character 86 [1], convergent in the brachylophosaurine clade), invaginated caudal region of the circumnarial fossa (character 171 [2]), and absence supracranial crest (character 173 [0], convergent in Acristavus and non-Saurolophidae Hadrosauriformes except Lophorhothon).

Theposition of L. youngi withinEdmontosaurini is not well resolved, but this taxon can still be distinguished from others in this clade on the basis of seven autapomorphies: 33 to 44 tooth positions in the maxillary dental battery (character 11 [1]), maxillary battery contains a mixture of teeth with primary ridge positioned caudally and teeth with the ridge at the center of the crown (character 14 [0]), retroarticular process of the surangular laterally recurved (character 43 [1]), one or more foramina on the rostral surface of the premaxilla (character 61 [1]), relatively shallow dorsoventral expansion of the caudal margin of the lacrimal process of the jugal, rostrodorsally directed and forming little of the rostroventral margin of the orbital rim (character 97 [0]), medial articular surface of the rostral process of the jugal deep concavity roofed by an oblique (rostrodorsally oriented) narrow shelf (character 100 [1]), and a wider orbital margin and relatively constricted ventral margin of the infratemporal fenestra (character 106 [2]).

\section{CONCLUSIONS}

Laiyangosaurus youngi is a new taxon of saurolophine dinosaurid from Jingangkou Formation, Wangshi Group, Laiyang City, Shandong Province, China. Laiyangosaurus shows typical saurolophine morphologies, and they can be easily distinguished from the equivalent bones of the other two Laiyang hadrosauroids, the non-hadrosaurid hadrosauroid Tanius and the Lambeosaurine Tsintaosaurus. Although Laiyangosaurus share some important features with the saurolophine Shantungosaurus, which was found from Zhucheng and Laiyang, there are a number of significant differences between them, and Laiyangosaurus does not represent a juvenile form of Shantungosaurus. The phylogenetic analysis indicates that Laiyangosaurus lies in a monophyletic group, which is known as Edmontosaurini. This is in consistent with the result of our anatomical comparison. The establishment of the new saurolophine hadrosaurid, L. youngi, further clarifies composition of the Laiyang Hadrosauroid Fauna.

\section{ACKNOWLEDGMENTS}

We thank Yan Li, Long Xiang, Wei Gao, Hongjiao Zhou, Ruijie Wang, Xinjun Zhang, Rui Pan, Dan $\mathrm{Xu}$, Wenhao Wang, and Huashuan Shou (IVPP) for excavating and preparing the specimens described here, as well as Wei Gao for taking some of the photos that illustrate this article. We also thank Guoqiang Yang, Shenggang Li, Changhou Zou, Mengxuan Lu, Xinzhang Gai, Hong Lan, Yan Wang, Weihua Zhang, Yu Sun, Yuting Zhao, Wenbin Li, Jian Li and Jianhua Wang (Laiyang) for supporting our excavations. We also thank Gareth Dyke (Department of Evolutionary Zoology, University of Debrecen, Hungary) and two reviewers for their valuable comments and English correction on the manuscript. This study was supported by the National Natural Science Foundation of China (41172018, 41688103, 41572020), the Major Research plan of the National Natural Science Foundation of China (91514302), the Strategic Priority Research Program (B) of the Chinese Academy of Sciences (XDB18000000), the National Key Basic Research Program of China 
(2012CB821900), the National Science Fund for Distinguished Young Scholars (40825005), and the Laiyang Government Cooperation Dinosaur Project.

\section{REFERENCES}

BELL PR. 2011. Cranial osteology and ontogeny of Saurolophus angustirostris from the Late Cretaceous of Mongolia with comments on Saurolophus osborni from Canada. Acta Palaeontol Pol 56(4): 703-722.

BOLOTSKY Y AND GODEFROIT P. 2004. A new hadrosaurine dinosaur from the Late Cretaceous of Far Eastern Russia. J Vert Palaeontol 24: 354-368.

BRETT-SURMAN MK. 1979 Phylogeny and palaeobiogeography of hadrosaurian dinosaurs. Nature 277: 560-562.

BROWN B. 1910. The Cretaceous Ojo Alamo beds of New Mexico with description of the new dinosaur genus Kritosaurus. Bull Am Mus Nat Hist 28: 267-274.

BROWN B. 1912. A crested dinosaur from the Edmonton Cretaceous. Bull Am Mus Nat Hist 31: 131-136.

BROWN B. 1914. Corythosaurus casuarius, a new crested dinosaur from the Belly River Cretaceous, with provisional classification of the family Trachodontidae. Bull Am Mus Nat Hist 33: 559-565.

BROWN B. 1916. A new crested trachodont dinosaur Prosaurolophus maximus. Bull Am Muse Nat Hist 35: 701-708.

BUFFETAUT E AND TONG HY. 1995. The Late Cretaceous dinosaurs of Shandong, China: old finds and new interpretations. In: Sun AL and Wang YQ (Eds), Sixth Symposium on Mesozoic Terrestrial Ecosystems and Biota, Beijing: China Ocean Press, p. 139-142.

COPE ED. 1869. Synopsis of the extinct Batrachia, Reptilia, and Aves of North America. Trans Am Philos Soc 14: 1-252.

DONG ZM. 1978. A new genus of Pachycephalosauria from Laiyang, Shantung. Vert PalAsiat 16(4): 225-228.

FOWLER EAF AND HORNER JR. 2015. A New Brachylophosaurin Hadrosaur (Dinosauria: Ornithischia) with an Intermediate Nasal Crest from the Campanian Judith River Formation of Northcentral Montana. PloS ONE 10(11): e0141304.

GATES TA, HORNER JR, HANNA RR AND NELSON CR. 2011. New unadorned hadrosaurine hadrosaurid (Dinosauria, Ornithopoda) from the Campanian of North America. J Vert Paleont 31(4): 798-811.

GATES TA AND SAMPSON SD. 2007. A new species of Gryposaurus (Dinosauria: Hadrosauridae) from the late Campanian Kaiparowits Formation, southern Utah, USA. Zool J Linn Soc 151(2): 351-376.

GATES TA AND SCHEETZ R. 2014. A new saurolophine hadrosaurid (Dinosauria: Ornithopoda) from the
Campanian of Utah, North America. J Syst Palaeontol 13(8): 711-725.

GILMORE CW. 1933. On the dinosaurian fauna of the Iren Dabasu Formation. Bull Am Mus Nat Hist 67: 23-78.

GODEFROIT P, BOLOTSKY YL AND LAUTERS P. 2012. A new saurolophine dinosaur from the latest Cretaceous of Far Eastern Russia. PloS ONE 7(5): e36849.

GODEFROIT P, HAI SL, YU TX and LAUTERS P. 2008. New hadrosaurid dinosaurs from the uppermost Cretaceous of northeastern China. Acta Palaeontol Pol 53: 47-74.

GOLOBOFF PA, FARRIS JS AND NIXON K. 2008. TNT, a free program for phylogenetic analysis. Cladistics 24(5): 774-786.

HORNER JR. 1992. Cranial morphology of Prosaurolophus (Ornithischia: Hadrosauridae) with descriptions of two new hadrosaurid species and an evaluation of hadrosaurid phylogenetic relationships. Mus Rock Occas Pap 2: 1-119.

HORNER JR AND MAKELA R. 1979. Nest of juveniles provides evidence of family structure among dinosaurs. Nature 282: 296-298.

HORNER JR, WEISHAMPEL DB AND FORSTER CA. 2004. Hadrosauridae. In: Weishampel DB, Dodson P and Osmólska H (Eds), The Dinosauria, $2^{\text {nd }}$ ed., Berkeley: University of California Press, p. 438-463.

HOPSON JA. 1975. The evolution of cranial display structures in hadrosaurian dinosaurs. Paleobiology 1: 21-43.

HU CZ. 1973. A new hadrosaur from the Cretaceous of Zhucheng, Shantung. Acta Geol Sin 2: 179-202.

HU CZ, CHENG ZW PANG QQ AND FANG XS. 2001. Shantungosaurus giganteus, Beijing: Geological Publishing House, $116 \mathrm{p}$.

JUÁREZ VALIERI RD, HARO JA, FIORELLI LE AND CALVO JO. 2010. A new hadrosauroid (Dinosauria: Ornithopoda) from the Allen Formation (Late Cretaceous) of Patagonia, Argentina. Rev Mus Argenti Cienc Nat 12(2): 217-231.

LAMBE LM. 1914. On Gryposaurus notabilis, a new genus and species of trachodont dinosaur from the Belly River Formation of Alberta, with description of the skull of Chasmosaurus belli. Ott Nat 27: 145-155.

LAMBE LM. 1917. A new genus and species of crestless hadrosaur from the Edmonton Formation of Alberta. Ott Nat 31: 65-73.

LUND EK AND GATES TA. 2006. A historical and biogeographical examination of hadrosaurian dinosaurs. New Mexico Mus Nat Hist Sci Bull 35: 263-276.

MARSH OC. 1881. Principal characters of American Jurassic dinosaurs. Part IV. Am J Sci, Ser 3 21: 417-423.

MARSH OC. 1892. Notice of new reptiles from the Laramie Formation. Am J Sci Ser 3 257: 449-453.

MATEUS O, POLCYN MJ, JACOBS LL, ARAÚJO R, SCHULP AS, MARINHERIO J, PEREIRA B AND VINEYARD D. 2012. Cretaceous amniotes from Angola: 
dinosaurs, pterosaurs, mosasaurs, plesiosaurs, and turtles. Actas de V Jornadas Internacionales sobre Paleontología de Dinosaurios y su Entorno, Salas de los Infantes, Burgos, p. 71-105.

MCGARRITY CT, CAMPIONE NE AND EVANS DC. 2013. Cranial anatomy and variation in Prosaurolophus maximus (Dinosauria: Hadrosauridae). Zool J Linn Soc 167(4): 531568.

MORI H, DRUCKENMILLER PS AND ERICKSON GM. 2015. A new Arctic hadrosaurid from the Prince Creek Formation (lower Maastrichtian) of northern Alaska. Acta Palaeontol Pol 61(1): 15-32.

NORMAN DB. 2004. Basal Iguanodontia. In: Weishampel DB, Dodson P and Osmólska H (Eds), The Dinosauria, $2^{\text {nd }}$ ed., Berkeley: University of California Press, p. 413-437.

OSTROM JH. 1962. The cranial crests of hadrosaurian dinosaurs. Postilla 62: 1-29.

PRIETO-MÁRQUEZ A. 2008. Phylogeny and historical biogeography of hadrosaurid dinosaurs. $\mathrm{PhD}$ dissertation, Florida State University, Tallahassee, Florida, 936 p.

PRIETO-MÁRQUEZ A. 2010a. Global historical biogeography of hadrosaurid dinosaurs. Zool J Linn Soc 159: 530-525.

PRIETO-MÁRQUEZ A. 2010b. Global phylogeny of Hadrosauridae (Dinosauria: Ornithopoda) using parsimony and Bayesian methods. Zool J Linn Soc 159: 435-502.

PRIETO-MÁRQUEZ A. 2011. The skull and appendicular skeleton of Gryposaurus latidens, a saurolophine hadrosaurid (Dinosauria: Ornithopoda) from the early Campanian (Cretaceous) of Montana, USA. Can J Earth Sci 49: 510-532.

PRIETO-MÁRQUEZ A. 2014. A juvenile Edmontosaurus from the late Maastrichtian (Cretaceous) of North America: Implications for ontogeny and phylogenetic inference in saurolophine dinosaurs. Cretaceous Res 50: 282-303.

PRIETO-MÁRQUEZ A, ERICKSON GM AND EBERSOLE JA. 2016. A primitive hadrosaurid from southeastern North America and the origin and early evolution of 'duckbilled'dinosaurs. J Vert Paleont 36(2): e1054495.

PRIETO-MARQUEZ A AND SALINAS GC. 2010. A ReEvaluation of Secernosaurus koerneri and Kritosaurus australis (Dinosauria, Hadrosauridae) from the Late Cretaceous of Argentina. J Vert Paleont 30(3): 813-837.

ROZHDESTVENSKY AK. 1952. A new representative of duckbilled dinosaurs from the Upper Cretaceous deposits of Mongolia. Dokl Akad SSSR 86: 405-408.

SEELEY HG. 1887. On the classification of the fossil animals commonly named Dinosauria. Proc R Soc London 43: $165-171$.

STERNBERG CM. 1926. A new species of Thespesius from the Lance Formation of Saskatchewan. Can Dep Mines Bull Geol Ser 44: 73-84.
STERNBERG CM. 1953. A new hadrosaur from the Oldman Formation of Alberta: discussion of nomenclature. Natl Mus Can Bull 128: 275-286.

TAN XC. 1923. New research on the Mesozoic and early Tertiary geology in Shantung. Bull Geol Surv China 5(2): 95-135

TAQUET P. 1976. Ostéologie d'Ouranosaurus nigeriensis, Iguanodontide du Crétacé Inférieur du Niger. Géologie et Paléontologie du Gisement de Gadoufaoua (Aptien du Niger), Chapitre 3: 57-168.

WANG Q, WANG XL, ZHAO ZK, ZHANG JL AND JIANG SX. 2013a. New turtle egg fossil from the Upper Cretaceous of the Laiyang Basin, Shandong Province, China. An Acad Bras Cienc 85:103-111.

WANG RF, YOU HL, XU SC, WANG SZ, YI J, XIE LJ, JIA L AND LI YX. 2013b. A new hadrosauroid dinosaur from the early Late Cretaceous of Shanxi Province, China. PloS ONE 8(10): e77058.

WANG XL, WANG Q, JIANG SX, CHEN X, ZHANG JL, ZHAO ZK AND JIANG YG. 2012. Dinosaur egg faunas of the Upper Cretaceous terrestrial red beds of China and their stratigraphical significance. J Stratigr 36(2): 400-416.

WANG XL, WANG Q, WANG JH, ZHANG JL, CHENG X, JIANG SX AND PAN R. 2010. An overview on the Cretaceous dinosaurs and their eggs from Laiyang, Shandong Province, China. In: Dong W (Eds), Proceedings of the Twelfth Annual Meeting of the Chinese Society of Vertebrate Paleontology, Beijing: China Ocean Press, p. 293-316.

WIMAN C. 1929. Die Kreide-Dinosaurier aus Shantung. Paleont Sin Ser C 6(1): 1-67.

XING H, PRIETO-MÁRQUEZ A, GU W AND YU TX. 2012. Reevaluation and phylogenetic analysis of the hadrosaurine dinosaur Wulagasaurus dongi from the Maastrichtian of northeast China. Vert PalAsiat 50(2): 160-169.

XING H, WANG DY, HAN FL, SULLIVAN C, MA QY, HE YM, HONE DWE, YAN RH, DU FM AND XU X. 2014a. A new basal hadrosauroid dinosaur (Dinosauria: Ornithopoda) with transitional features from the Late Cretaceous of Henan Province, China. PLoS ONE 9(6): e98821.

XING H, ZHAO XJ, WANG KB, LI DJ, CHEN SQ, MALLON JC, ZHANG YX AND XING X. 2014b. Comparative Osteology and Phylogenetic Relationship of Edmontosaurus and Shantungosaurus (Dinosauria: Hadrosauridae) from the Upper Cretaceous of North America and East Asia. Acta Geol Sin-Engl 88(6): 1623-1652.

YAN J AND CHEN JF. 2005. Clinopyroxene megacrysts in the Late Mesozoic basalts from Daxizhuang, Jiaozhou. J Anhui Univ Sci Technol (Nat Sci) 25(3): 9-13.

YOU HL, LUO ZX, SHUBIN NH, WITMER LM, TANG ZL AND TANG F. 2003. The earliest-known duck-billed dinosaur from deposits of late Early Cretaceous age in Northwest China and hadrosaur evolution. Cretaceous Res 24: 347-355. 
YOUNG CC. 1958. The dinosaurian remains of Laiyang, Shantung. Palaeontol Sin, New Ser C16: 1-138.

ZHEN SN. 1976. A new species of hadrosaur from Shandong. Vert Palasiat 14(3): 166-168.

\section{APPENDIX A}

Character state scores of L. youngi for the 273 characters presented by Prieto-Márquez (2016). The scores for all the other taxa included in the analysis are as in Prieto-Márquez (2016).
[01]??0??????11102??????????1[01]01100110211 10011????31?????????0?10-?00???-??-?11?[01]22 0211112321-00??1000110?010????????????????? ???2??????????????????????????????1?1?-??--01 112-00------?????????????????????????????????? ??????????????????????????????????????????? ???????????? 